\title{
A INFRAESTRUTURA DAS ESCOLAS DE ENSINO FUNDAMENTAL DA REGIÃO DO GRANDE ABC PAULISTA
}

\author{
Paulo Sérgio GARCIA ${ }^{1}$ \\ Leandro Campi PREARO ${ }^{2}$ \\ Maria do Carmo ROMERO ${ }^{3}$ \\ Marcos Sidnei BASSI ${ }^{4}$
}

RESUMO: No Brasil, o sistema de avaliação educacional procura investigar e compreender os fatores que influenciam o desempenho dos alunos em testes de larga escala para, com tal entendimento, lançar políticas para melhorar da educação brasileira. Um desses fatores é a infraestrutura das escolas que no cenário brasileiro influencia a aprendizagem dos alunos. Este estudo investiga, explorando, identificando e descrevendo, a infraestrutura das escolas de ensino fundamental da Região do Grande ABC Paulista, coletando informações a partir do Censo escolar de 2013 e analisando-as sob a perspectiva quantitativa. Foram identificadas e analisadas 17 categorias (Água filtrada, Sanitário, Esgoto, Cozinha, Energia Elétrica, Laboratório de informática, Laboratório de Ciências, Biblioteca, TV, DVD, Acesso à Internet, Computadores, Sala de Diretoria, Sala de professores, Impressora, Copiadora e Quadra esportiva) e realizadas comparações com o cenário brasileiro. Os resultados mostraram que a infraestrutura escolar da Região está bem consolidada, superando de longe a dura realidade brasileira. Em uma escala desenvolvida por pesquisadores brasileiros para medir a infraestrutura das escolas, a Região está situada no nível avançado (74\%). A compreensão de tal realidade pode favorecer a reflexão sobre possíveis alternativas para a organização ou reformas do sistema educacional ou das escolas.

PALAVRAS-CHAVE: Infraestrutura. Escolas. Ensino Fundamental.

\section{Introdução}

Com avanços realizados nas décadas passadas quanto à democratização do acesso à escola básica, as novas discussões e políticas se deslocaram para a preocupação com a qualidade do ensino, que requer esforços multifacetados advindos do governo, dos especialistas e da sociedade. Tal preocupação com a qualidade do ensino, como

\footnotetext{
${ }^{1}$ Professor. USCS - Universidade Municipal de São Caetano do Sul. Departamento de Educação. São Caetano do Sul - SP - Brasil. 09550-051. Observatório da Educação do Grande ABC - garciaps@usp.br.

${ }^{2}$ USCS - Universidade Municipal de São Caetano do Sul. Departamento de Administração. São Caetano do Sul - SP - Brasil. 09550-051. Observatório da Educação do Grande ABC leandro.prearo@uscs.edu.br.

${ }^{3}$ USCS - Universidade Municipal de São Caetano do Sul. Departamento de Administração. São Caetano do Sul - SP - Brasil. 09550-051. Observatório da Educação do Grande ABC - mromeiro@uscs.edu.br. ${ }^{4}$ USCS - Universidade Municipal de São Caetano do Sul. Departamento de Administração. São Caetano do Sul - SP - Brasil. 09550-051. Observatório da Educação do Grande ABC-mbassi@uscs.
} 
marco jurídico-institucional, ocorreu a partir da Constituição Federal de 1988 (BRASIL, 1988), seguida da Lei de Diretrizes e Bases da Educação Nacional (LDBEN/96) (BRASIL, 1996) que procurou estabelecer um padrão de qualidade, entre outras coisas, mencionando os insumos e o cálculo do custo mínimo por aluno.

A atual Constituição Federal, instituindo o marco jurídico-institucional, no Art. 206, inciso VII, determinou a "garantia de padrão de qualidade" para a Educação. As modificações na ordem jurídico-institucional completaram-se com a aprovação de outros instrumentos legais que impactaram a Educação brasileira. Nesse processo, destacam-se: a Lei de Diretrizes e Bases da Educação Nacional (Lei n 9.394, de 1996 LDBEN), em seu artigo terceiro, inciso IX, que propôs a "garantia de padrão de qualidade" como princípio do ensino e definiu no Art. $4^{\circ}$, inciso IX, "padrões mínimos de qualidade de ensino, definidos como a variedade e quantidade mínimas, por aluno, de insumos indispensáveis ao desenvolvimento do processo de ensino-aprendizagem". Outros artigos desta legislação também fizeram referência à qualidade $\left(70^{\circ}, 71^{\circ}, 72^{\circ}, 74^{\circ}\right.$ e 75º); a Emenda Constitucional no 14, de 1996, que instituiu o Fundo de Manutenção e Desenvolvimento do Ensino Fundamental e de Valorização do Magistério (FUNDEF) com o objetivo de redistribuição dos recursos vinculados à Educação, visando cumprir o princípio constitucional da equalização do financiamento; a Lei $n^{\circ} 10.172$, de 2001, que estabeleceu o Plano Nacional de Educação (PNE), determinando novos caminhos para as políticas e ações do governo e fixando objetivos e metas para a Educação brasileira por um período de dez anos (chamada "Década da Educação"); a Lei n ${ }^{\circ} 11.494$, de 2007, que regulamentou o Fundo de Manutenção e Desenvolvimento da Educação Básica e de Valorização dos Profissionais da Educação (FUNDEB); a Emenda Constitucional $\mathrm{n}^{\mathrm{o}} 59$, de 2009, que amplia o ensino obrigatório dos quatro aos 17 anos de idade; e, recentemente, ainda rumo à qualidade de ensino, foi aprovado o novo Plano Nacional de Educação (PNE), Lei n. 13.005, 25 de junho de 2014, instituindo aos estados e municípios o prazo de um ano para que esses entes realizem também seus planos.

A preocupação com a qualidade do ensino no Brasil fez surgir também medidas que foram implantadas, entre outras, de coleta de informação, recenseamento escolar e verificação de desempenho acadêmico. Nas últimas décadas, vários instrumentos em nível nacional e estadual foram criados e inseridos na Educação brasileira, como é o caso do Sistema de Avaliação da Educação Básica (SAEB), de dezembro de 1994, 
Portaria n. 1.795, com o intuito de melhorar a qualidade da Educação e o desempenho dos alunos.

Os estudos sobre SAEB possibilitaram analisar dados em larga escala e, ao mesmo tempo, captar uma realidade de forma mais detalhada e profunda. Foi possível compreender que grande parte do desempenho acadêmico dos jovens brasileiros está relacionada à família (ao redor de dois terços), a um conjunto de variáveis agregadas, sobretudo a escolaridade dos pais e o nível socioeconômico (ALBERNAZ; FERREIRA; FRANCO, 2002; FELICIO; FERNANDES, 2005). A outra parte do desempenho, o efeito escola, dos adolescentes está associado às características da escola (FLETCHER, 1997; FERRÃO; BELTRÃO; SANTOS, 2002).

Apesar da compreensão, após a publicação do Relatório Coleman (1966), de que os fatores individuais (nível socioeconômico - NSE) explicam a maior porcentagem da variação observada entre as proficiências dos estudantes, mais do que os fatores escolares, como afirmam César e Soares (2001, p.97) “[...] o poder explicativo destes últimos fatores é suficientemente alto para alterar a trajetória escolar dos alunos. Assim sendo, os fatores escolares têm sido amplamente estudados na literatura".

As pesquisas brasileiras ligadas à avaliação educacional envidam esforços para entender as variáveis explicativas do desempenho escolar dos alunos em avaliações de larga escala para, a partir desse entendimento, desenvolver e lançar políticas públicas com o propósito de melhorar a qualidade da Educação. Neste sentido, vários fatores são responsáveis pelo desempenho escolar do aluno. Entre eles está a infraestrutura (IE) que na realidade brasileira é uma categoria que impacta o desempenho dos alunos.

O tema infraestrutura engloba as instalações, os equipamentos pedagógicos e os serviços realizados na escola. Trata-se de uma variável que influencia o desempenho escolar dos alunos, diferentemente do que ocorre em países desenvolvidos onde as escolas possuem, praticamente, os mesmos recursos tanto para o funcionamento como para a aprendizagem das crianças e dos jovens. No Brasil, a localização da escola, rural ou urbana, ou a esfera, municipal, estadual, particular, determina em grande parte a infraestrutura escolar.

No Brasil já está bem consolidado o fato de que o rendimento do aluno é tributário da infraestrutura. Vários estudos demonstraram tal afirmação. Na década de 1980, Castro e Fletcher (1986), em uma das primeiras pesquisas na área, identificaram a precária situação da IE das redes escolares. Os autores mostraram as más condições das 
instalações físicas e a pouca quantidade de equipamentos disponível para a realização das atividades pedagógicas.

Outros estudos também reforçaram a premissa da infraestrutura impactando positivamente o desempenho dos alunos em qualquer uma das cinco grandes regiões do Brasil (BARBOSA; FERNANDES, 2001). Também Albernaz, Ferreira e Franco (2002), utilizando dados do SAEB de 1999, da $8^{\text {a }}$ série, mostraram que a falta de recursos financeiros e pedagógicos impactam negativamente a eficácia da escola.

A partir de dados do SAEB 2001, $8^{a}$ série (INEP, 2015), Soares (2004) e Franco, Sztajn e Ortigão (2007) mostraram que a infraestrutura, a presença de equipamentos pedagógicos e a conservação desses na escola fazem diferença em relação à aprendizagem dos alunos. Lee, Franco e Albernaz (2004) demostraram que a IE teve efeito positivo sobre o desempenho em leitura dos alunos brasileiros que participaram do Programa Internacional de Avaliação de Estudantes PISA (2000). No mesmo sentido, outra pesquisa também reportou que a infraestrutura e os equipamentos são categorias importantes para o rendimento dos alunos (FRANCO, SZTAJN; ORTIGÃO, 2007).

Um estudo importante (SÁTYRO; SOARES, 2007) analisou as condições da infraestrutura das escolas do Ensino Fundamental, utilizando dados dos Censos Escolares de 1997 a 2005. A pesquisa mostrou que ocorreram melhorias na IE no período de 1997 a 2005, que, no entanto, não incidiram sobre a repetência e o aprendizado dos alunos. No caso das escolas rurais, por exemplo, essas apresentaram as piores condições de IE e de resultados educacionais. Na mesma direção, o estudo de Soares, Razo e Fariñas (2006) revelou que a infraestrutura escolar é um fator importante que explica, em muitos casos, os baixos resultados da Educação na área rural.

Espósito, Davis e Nunes (2000) mostraram resultados positivos para o efeito das condições de funcionamento de laboratórios e espaços adicionais para atividades pedagógicas. Franco e Bonamino (2005) revelaram que no Brasil e em outros países da América Latina, diferente de países desenvolvidos, a IE é fundamental para o rendimento escolar dos jovens, mesmo quando os resultados são controlados pelo nível socioeconômico dos alunos.

Vale ressaltar que a simples presença de uma biblioteca com livros adequados, de laboratórios ou de computadores na escola não garante a aprendizagem dos jovens. Neste caso, a questão central é a utilização desses espaços e recursos associados com iniciativas e projetos pedagógicos. A infraestrutura torna-se importante para a formação 
do jovem quando articulada com as instalações, os equipamentos, os serviços, as iniciativas educativas, os projetos pedagógicos e o comportamento humano.

Vale citar ainda o contexto internacional onde Hattie (2009), em uma análise do impacto da infraestrutura no desempenho dos estudantes, revelou que a maior fonte de variância reside dentro das escolas e não entre as escolas.

Recentemente, Soares Neto et al (2013) construíram uma escala, com a criação de níveis, para medir a infraestrutura no Brasil. Os níveis classificam as escolas no patamar Elementar: aquelas que possuíam aspectos elementares para o funcionamento (água, sanitário, energia, esgoto e cozinha); Básico, acrescido aos itens presentes no nível anterior, as escolas aqui possuíam sala de diretoria e equipamentos (TV, DVD, computadores e impressora); Adequado, as unidades escolares possuíam infraestrutura, possibilitando um ambiente mais propício para o ensino e aprendizagem (espaços como sala, de professores, biblioteca, laboratório de informática, sanitário para Educação Infantil, quadra esportiva, parque infantil, copiadora e acesso à internet); Avançado, os estabelecimentos possuíam uma IE mais próxima do ideal, contando com laboratórios de ciências e dependências adequadas para atender estudantes com necessidades especiais.

Neste cenário, o maior número de escolas estava localizado no nível Elementar nas regiões Norte $(71 \%)$ e Nordeste $(65,1 \%)$. O Sudeste contava com 22,7\% neste plano, 57\% no Básico, 19,8\% no Adequando e 0,5\% no Avançado. As escolas federais apresentaram os melhores resultados (62,5\% nos níveis Adequado e Avançado). Das estaduais, 51,3\% estavam na categoria Básica e 61,8\% das municipais na categoria Elementar.

Os autores demostraram que, quase, 84,5\% (164.786) das escolas estavam localizadas no nível Elementar ou Básico (45\% nível Elementar, 86.739; $40 \%$ no Básico, 78.047). O estudo mostrou também que somente $14,9 \%$ das escolas se encontravam no plano Adequado (29.026) e 0,6 no Avançado (1.120). Esses dados contribuíram para revelar o cenário empobrecido da realidade brasileira em relação à infraestrutura, onde quase $85 \%$ das escolas brasileiras possuem um nível Elementar ou Básico.

Considerando o cenário empobrecido da realidade brasileira e a premissa mostrada em vários estudos de que a infraestrutura influencia a aprendizagem dos alunos, este estudo investiga, explorando, identificando e descrevendo, a infraestrutura (instalações, equipamentos e os serviços necessários para garantir o funcionamento da 
escola e auxiliar na aprendizagem do aluno) das escolas de ensino fundamental da Região do Grande ABC Paulista. Paralelamente, são realizadas comparações com o cenário brasileiro.

As informações foram coletadas a partir do Censo escolar de 2013 e analisadas sob a perspectiva quantitativa. Neste sentido, pretende-se construir um cenário para a Região, contribuindo para o entendimento de possíveis alternativas para a organização ou reformas do sistema educacional ou das escolas.

\section{A Região do Grande ABC Paulista}

A região do Grande $\mathrm{ABC}$, formada por sete municípios: Santo André, São Bernardo do Campo, São Caetano do Sul, Diadema, Mauá, Ribeirão Pires e Rio Grande da Serra, está localizada no estado de São Paulo.

É uma região com mais de 2,5 milhões de habitantes e uma área de $635 \mathrm{~km}^{2}$ (IBGE, 2015). Ela tem um PIB Industrial de cerca de R \$ 27 bilhões, que é o segundo do estado e o terceiro do país. São 44 mil estabelecimentos formais, gerando $800 \mathrm{mil}$ empregos, mais ou menos 37 mil unidades comerciais e de serviços, com 530 mil empregos e 6,3 mil unidades industriais. (Consórcio Intermunicipal do Grande ABC, 2013).

O Quadro 1 mostra o índice de desenvolvimento humano municipal (IDHM) das cidades:

Quadro 1 - IDHM 2010

\begin{tabular}{|c|c|}
\hline Município & IDHM 2010 \\
\hline Diadema & 0,757 \\
\hline Mauá & 0,766 \\
\hline Santo André & 0,815 \\
\hline $\begin{array}{c}\text { São Bernardo do } \\
\text { Campo }\end{array}$ & 0,805 \\
\hline São Caetano do Sul & 0,862 \\
\hline Rio Grande da Serra & 0,749 \\
\hline Ribeirão Pires & 0,784 \\
\hline
\end{tabular}

Fonte: Adaptado pelo autor dos dados do Atlas de desenvolvimento humano - 2013. Disponível em: <http://www.atlasbrasil.org.br/2013/>. Acesso em: 25 fev. 2015. 
As cidades que foram analisadas apresentam características demográficas diferentes. O Quadro 2 sintetiza esses atributos:

Quadro 2 - Características demográficas

\begin{tabular}{|c|c|c|c|c|}
\hline Cidade & População & Área territorial Km2 & PIB (milhões) & Posição do PIB \\
\hline Rio Grande da Serra & 47.142 & 36,341 & 529.413 & $816^{\mathrm{a}}$ \\
\hline Ribeirão Pires & 118.871 & 99,119 & 1.978 .256 & $287^{\mathrm{a}}$ \\
\hline Mauá & 444.136 & 61,866 & 7.633 .782 & $79^{\mathrm{a}}$ \\
\hline Diadema & 406.718 & 30,796 & 11.786 .624 & $47^{\mathrm{a}}$ \\
\hline São Caetano do Sul & 156.362 & 15,33 & 11.762 .744 & $48^{\mathrm{a}}$ \\
\hline Santo André & 704.942 & 175,781 & 17.664 .718 & $33^{\mathrm{a}}$ \\
\hline São Bernardo do Campo & 805,895 & 409,478 & 36.337 .338 & $14^{\mathrm{a}}$ \\
\hline
\end{tabular}

Fonte: Elaboração própria com base em IBGE (2015).

Pode-se verificar que as cidades maiores em área, São Bernardo e Santo André, são também os municípios de maior PIB na Região.

\section{Metodologia}

Este estudo tem como objetivo investigar, explorando, identificando e descrevendo, a infraestrutura (instalações, equipamentos e os serviços necessários para garantir o funcionamento da escola e auxiliar na aprendizagem do aluno) das escolas de ensino fundamental da Região do Grande ABC Paulista. Paralelamente, são realizadas comparações com o cenário brasileiro. A intenção desta pesquisa é realizar um estudo puramente descritivo, quantitativo, com algumas pretensões analíticas. Não é parte do propósito nesta pesquisa correlacionar o desempenho dos alunos e a infraestrutura das escolas.

A Região do Grande ABC Paulista foi selecionada, pois acredita-se que ela apresenta uma realidade diferente e difícil de ser encontrada nas outras regiões do país.

Os dados foram coletados a partir do Censo de Educação de 2013, microdados Censo Escolar, no site do Instituto Nacional de Estudos e Pesquisas Educacionais Anísio Teixeira (INEP). O estudo, baseado na metodologia quantitativa ideal para experimentos, para análise de dados em larga escala, testar hipóteses, quantificar, comparar e criar relações, analisou tanto a esfera municipal como estadual.

As análises foram realizadas, utilizando o software para o uso estatístico Statistical Package for the Social Sciences (SPSS) versão 19. Foram identificadas 17 
categorias: Água filtrada, Sanitário, Esgoto, Cozinha, Energia Elétrica, Laboratório de informática, Laboratório de Ciências, Biblioteca, TV, DVD, Acesso à Internet, Computadores, Sala de Diretoria, Sala de professores, Impressora, Copiadora e Quadra esportiva.

Os dados são apresentados em forma de tabelas, onde são mostradas as proporções de cada componente da infraestrutura por município, no Grande ABC e no Brasil, tanto na esfera municipal como estadual.

A partir do estudo realizado por Soares Neto et al (2013), as escolas do Grande $\mathrm{ABC}$ foram classificadas quanto à infraestrutura. Foram também realizadas comparações com a realidade brasileira, no intuito de perceber as relações.

\section{Resultados e discussão}

Na realidade brasileira, dados de 2013 mostraram que existiam 190.706 escolas, sendo dessas 512 abrigadas na esfera federal, 30.891 na estadual, 120.481 na municipal e 38.822 na esfera privada. No ensino fundamental, as escolas totalizavam 141.260 unidades, com 45 federais, 25.003 estaduais, 93.866 municipais e 22.346 pertencentes à esfera privada.

A região do Grande $\mathrm{ABC}$, contava no mesmo ano, com 1.331 escolas, representando mais ou menos $0,7 \%$ do total das escolas brasileiras. No Ensino Fundamental do município eram 198 estabelecimentos de ensino e 327 do estado.

No Brasil, 50.042.448 de estudantes estavam matriculados nas escolas de Educação Básica no ano de 2013. No Ensino Fundamental 29.069.281 alunos. Na região do Grande ABC, as escolas de Ensino Fundamental possuíam 160.680 matrículas na esfera municipal e 273.054 na estadual.

A Região do Grande ABC está bem consolidada em relação à água filtrada, sanitário e esgoto na rede pública (Tabela 1) e também quanto a ter cozinha e energia elétrica (Tabela 2). Situação um pouco diversa da realidade brasileira, sobretudo aquela associada ao esgoto tanto da rede municipal $(22,8 \%)$ como estadual $(55,2 \%)$.

Em relação à água filtrada, sanitário e esgoto na rede pública, a Tabela 01 sintetiza os resultados: 
Tabela 1 - Água filtrada, sanitário e esgoto na rede pública - 2013.

\begin{tabular}{|l|l|l|l|l|l|l|l|l|}
\hline SA & SBC & SCS & DIA & MAU & RP & RGS & GABC & BRA \\
\hline & & & & & & & & \\
\hline 100,0 & 100,0 & 100,0 & 100,0 & 100,0 & 100,0 & 100,0 & $\mathbf{1 0 0 , 0}$ & $\mathbf{8 5 , 4}$ \\
\hline 98,0 & 98,6 & 100,0 & 100,0 & 87,5 & 100,0 & 66,7 & $\mathbf{9 7 , 5}$ & $\mathbf{9 3 , 3}$ \\
\hline 98,0 & 91,7 & 100,0 & 100,0 & 100,0 & 100,0 & 100,0 & $\mathbf{9 6 , 5}$ & $\mathbf{2 2 , 8}$ \\
\hline
\end{tabular}

Municipal \%

\begin{tabular}{|l|l|l|l|l|l|l|l|l|l|}
\hline Água filtrada & 100,0 & 100,0 & 100,0 & 100,0 & 100,0 & 100,0 & 100,0 & $\mathbf{1 0 0 , 0}$ & $\mathbf{8 5 , 4}$ \\
\hline Sanitário & 98,0 & 98,6 & 100,0 & 100,0 & 87,5 & 100,0 & 66,7 & $\mathbf{9 7 , 5}$ & $\mathbf{9 3 , 3}$ \\
\hline Esgoto (rede pública) & 98,0 & 91,7 & 100,0 & 100,0 & 100,0 & 100,0 & 100,0 & $\mathbf{9 6 , 5}$ & $\mathbf{2 2 , 8}$ \\
\hline Estadual \% & & & & & & & & & \\
\hline Água filtrada & 100,0 & 100,0 & 100,0 & 100,0 & 100,0 & 96,6 & 90,9 & $\mathbf{9 9 , 4}$ & $\mathbf{8 7 , 8}$ \\
\hline Sanitário & 97,6 & 98,6 & 100,0 & 96,4 & 100,0 & 100,0 & 100,0 & $\mathbf{9 8 , 5}$ & $\mathbf{9 7 , 0}$ \\
\hline Esgoto (rede pública) & 98,8 & 91,8 & 100,0 & 98,2 & 98,4 & 79,3 & 81,8 & $\mathbf{9 4 , 8}$ & $\mathbf{5 5 , 2}$ \\
\hline
\end{tabular}

Fonte: Elaboração própria com base nos dados do Censo de 2013 (INEP, 2015).

Quanto à escola ter cozinha e energia elétrica, a Tabela 2 sintetiza os resultados:

Tabela 2 - Cozinha e energia elétrica - 2013.

\begin{tabular}{|c|c|c|c|c|c|c|c|c|c|}
\hline & \multicolumn{7}{|c|}{ Município } \\
\hline Municipal & SA & SBC & SCS & DIA & MAU & RP & RGS & GABC & BRA \\
\hline Cozinha & 100,0 & 100,0 & 95,0 & 100,0 & 100,0 & 100,0 & 100,0 & $\mathbf{9 9 , 5}$ & $\mathbf{9 2 , 2}$ \\
\hline Energia Elétrica & 100,0 & 100,0 & 100,0 & 100,0 & 100,0 & 100,0 & 100,0 & $\mathbf{1 0 0 , 0}$ & $\mathbf{8 8 , 6}$ \\
\hline Estadual & & & & & & & & & \\
\hline Cozinha & 96,4 & 94,5 & 100,0 & 98,2 & 98,4 & 96,6 & 100,0 & $\mathbf{9 6 , 9}$ & $\mathbf{9 6 , 3}$ \\
\hline Energia Elétrica & 100,0 & 100,0 & 100,0 & 100,0 & 100,0 & 100,0 & 100,0 & $\mathbf{1 0 0 , 0}$ & $\mathbf{9 6 , 8}$ \\
\hline
\end{tabular}

Fonte: Elaboração própria com base nos dados do Censo de 2013 (INEP, 2015).

As escolas do Grande $\mathrm{ABC}$, em geral, contam com laboratórios de informática. Tal situação, no entanto, contrasta com a realidade brasileira, sobretudo aquela das instituições municipais. Neste quesito, os estabelecimentos de ensino estaduais estão mais equipados.

As escolas também dispõem de bibliotecas, apesar de os dados mostrarem certo desequilíbrio nesta categoria. Na esfera municipal, as unidades de ensino possuem mais esses espaços. A situação é um pouco mais difícil em relação aos laboratórios de 
ciências. A região ainda precisa avançar. Isto porque estudos brasileiros já mostraram que a utilização dos laboratórios de ciências e da biblioteca, componentes da infraestrutura, são determinantes para o desempenho escolar dos alunos (ESPÓSITO, DAVIS; NUNES, 2000; MENEZES FILHO, 2007; BIONDI; FELÍCIO, 2007).

Em relação a laboratório de informática, de ciências e biblioteca, as Tabelas 3, 4 e 5 mostram resultados:

Tabela 3 - Laboratório de informática - 2013.

\begin{tabular}{|c|c|c|c|c|c|c|c|c|c|}
\hline & \multicolumn{7}{|c|}{ Município } \\
\hline SA & SBC & SCS & DIA & MAU & RP & RGS & GABC & BRA \\
\hline Municipal & 100,0 & 97,2 & 90,0 & 63,0 & 93,8 & 88,9 & 0,0 & $\mathbf{9 0 , 4}$ & 41,6 \\
\hline Estadual & 94,0 & 93,2 & 90,0 & 87,5 & 84,4 & 82,8 & 72,7 & $\mathbf{8 9 , 0}$ & 82,9 \\
\hline
\end{tabular}

Fonte: Elaboração própria com base nos dados do Censo de 2013 (INEP, 2015).

Tabela 4 - Laboratório de ciências - 2013.

\begin{tabular}{|c|c|c|c|c|c|c|c|c|c|}
\hline & \multicolumn{7}{|c|}{ Município } \\
\hline SA & SBC & SCS & DIA & MAU & RP & RGS & GABC & BRA \\
\hline Municipal & 3,9 & 1,4 & 45,0 & 0,0 & 6,3 & 0,0 & 0,0 & $\mathbf{6 , 6}$ & $\mathbf{3 , 0}$ \\
\hline Estadual & 45,2 & 54,8 & 80,0 & 19,6 & 18,8 & 10,3 & 18,2 & $\mathbf{3 4 , 9}$ & $\mathbf{2 7 , 7}$ \\
\hline
\end{tabular}

Fonte: Elaboração própria com base nos dados do Censo de 2013 (INEP, 2015).

Tabela 5 - Biblioteca - 2013.

\begin{tabular}{|c|c|c|c|c|c|c|c|c|c|} 
& \multicolumn{7}{|c|}{ Município } \\
\hline & SA & SBC & SCS & DIA & MAU & RP & RGS & GABC & BRA \\
\hline Municipal & 60,8 & 97,2 & 45,0 & 40,7 & 18,8 & 11,1 & 0,0 & $\mathbf{6 3 , 1}$ & $\mathbf{2 3 , 0}$ \\
\hline Estadual & 16,7 & 9,6 & 10,0 & 3,6 & 26,6 & 20,7 & 18,2 & $\mathbf{1 5 , 0}$ & $\mathbf{6 1 , 1}$ \\
\hline
\end{tabular}

Fonte: Elaboração própria com base nos dados do Censo de 2013 (INEP, 2015).

A região também avançou em relação à presença de TV e DVD nas escolas, tecnologias que podem potencializar atividades pedagógicas, possibilitando a aquisição de conhecimento e o desenvolvimento de novas competências. No Brasil, na esfera municipal, a realidade ainda está um pouco defasada.

A questão dos computadores nas escolas parece ser um ponto já resolvido nas escolas estaduais, municipais e em todo o país. O acesso à internet, por outro lado, está 
um pouco mais consolidado nas escolas das redes municipais, porém na realidade brasileira as diferenças ainda são enormes, sobretudo aquela relativa às escolas municipais. Somente, aproximadamente, $30 \%$ das instituições brasileiras contam com acesso. A presença dos computadores e o acesso à rede sozinhas não são capazes de revolucionar as formas de aprendizagem, entretanto quando essas tecnologias são combinadas com novas pedagogias que incluem, entre outras coisas, parcerias entre alunos e professores os resultados alcançados podem ser significativos (FULLAN, 2013).

Em relação a ter DVD e TV e acesso à internet e computadores, as Tabelas 6 e 7 sintetizam os resultados:

Tabela 6 - TV e DVD - 2013.

\begin{tabular}{|c|c|c|c|c|c|c|c|c|c|}
\hline & \multicolumn{9}{|c|}{ Município } \\
\hline & SA & SBC & SCS & DIA & MAU & RP & RGS & GABC & BRA \\
\hline \multicolumn{10}{|c|}{ Municipal } \\
\hline TV & 100,0 & 97,2 & 90,0 & 96,3 & 100,0 & 100,0 & 100,0 & 97,5 & 64,5 \\
\hline DVD & 100,0 & 97,2 & 90,0 & 100,0 & 93,7 & 100,0 & 100,0 & 97,5 & 61,6 \\
\hline \multicolumn{10}{|c|}{ Estadual } \\
\hline TV & 98,8 & 100,0 & 90,0 & 98,2 & 95,3 & 100,0 & 90,9 & 97,9 & 92,0 \\
\hline DVD & 96,4 & 97,3 & 90,0 & 96,4 & 98,4 & 100,0 & 81,8 & 96,6 & 89,6 \\
\hline
\end{tabular}

Fonte: Elaboração própria com base nos dados do Censo de 2013 (INEP, 2015).

Tabela 7 - Acesso à internet e computadores - 2013.

\begin{tabular}{|c|c|c|c|c|c|c|c|c|c|}
\hline & \multicolumn{7}{|c|}{ Município } \\
\hline Municipal & SA & SBC & SCS & DIA & MAU & RP & RGS & GABC & BRA \\
\hline Acesso à internet & 90,2 & 97,2 & 90,0 & 92,6 & 93,8 & 66,7 & 100,0 & $\mathbf{9 2 , 4}$ & $\mathbf{2 9 , 4}$ \\
\hline Computadores & 100,0 & 100,0 & 100,0 & 100,0 & 100,0 & 100,0 & 100,0 & $\mathbf{1 0 0 , 0}$ & $\mathbf{1 0 0 , 0}$ \\
\hline Estadual & & & & & & & & & \\
\hline Acesso à internet & 83,3 & 89,0 & 70,0 & 83,9 & 78,1 & 75,9 & 63,6 & $\mathbf{8 2 , 0}$ & $\mathbf{7 3 , 5}$ \\
\hline Computadores & 100,0 & 100,0 & 100,0 & 100,0 & 100,0 & 100,0 & 100,0 & $\mathbf{1 0 0 , 0}$ & $\mathbf{1 0 0 , 0}$ \\
\hline
\end{tabular}

Fonte: Elaboração própria com base nos dados do Censo de 2013 (INEP, 2015). 
Em algumas cidades existem escolas que ainda não possuem sala de diretoria e de professores, muito embora em geral a região conte com tais locais. Muitas vezes, essas salas são adaptadas. Na esfera municipal, em termos de Brasil, a realidade é mais dura com falta desses espaços.

Dois recursos adicionais que auxiliam na preparação de atividades pedagógicas são a impressora e a copiadora. A primeira bem presente na realidade do Grande ABC, tanto nas escolas estaduais como municipais. Já a segunda, nem tanto. Há cidades, na esfera municipal (Diadema, Mauá e Rio Grande da Serra) em que ela não está tão presente. $\mathrm{Na}$ esfera estadual, a copiadora também não é um item presente em todas as escolas.

Em relação a ter sala de diretoria e de professores, as Tabelas 8 e 9 sintetizam os resultados:

Tabela 8 - Sala de diretoria e professores - 2013.

\begin{tabular}{|c|c|c|c|c|c|c|c|c|c|}
\hline & \multicolumn{7}{|c|}{ Município } \\
\hline Municipal & SA & SBC & SCS & DIA & MAU & RP & RGS & GABC & BRA \\
\hline Sala de Diretoria & 100,0 & 98,6 & 90,0 & 100,0 & 68,8 & 100,0 & 0,0 & $\mathbf{9 4 , 4}$ & $\mathbf{4 9 , 4}$ \\
\hline Sala de professores & 98,0 & 91,7 & 95,0 & 100,0 & 75,0 & 66,7 & 0,0 & $\mathbf{9 0 , 9}$ & $\mathbf{3 9 , 4}$ \\
\hline Estadual & & & & & & & & & \\
\hline Sala de Diretoria & 98,8 & 95,9 & 100,0 & 96,4 & 98,4 & 96,6 & 90,9 & $\mathbf{9 7 , 2}$ & $\mathbf{8 6 , 7}$ \\
\hline Sala de professores & 98,8 & 97,3 & 100,0 & 100,0 & 98,4 & 96,6 & 81,8 & $\mathbf{9 7 , 9}$ & $\mathbf{8 5 , 0}$ \\
\hline
\end{tabular}

Fonte: Elaboração própria com base nos dados do Censo de 2013 (INEP, 2015).

Tabela 9 - Impressora e copiadora - 2013

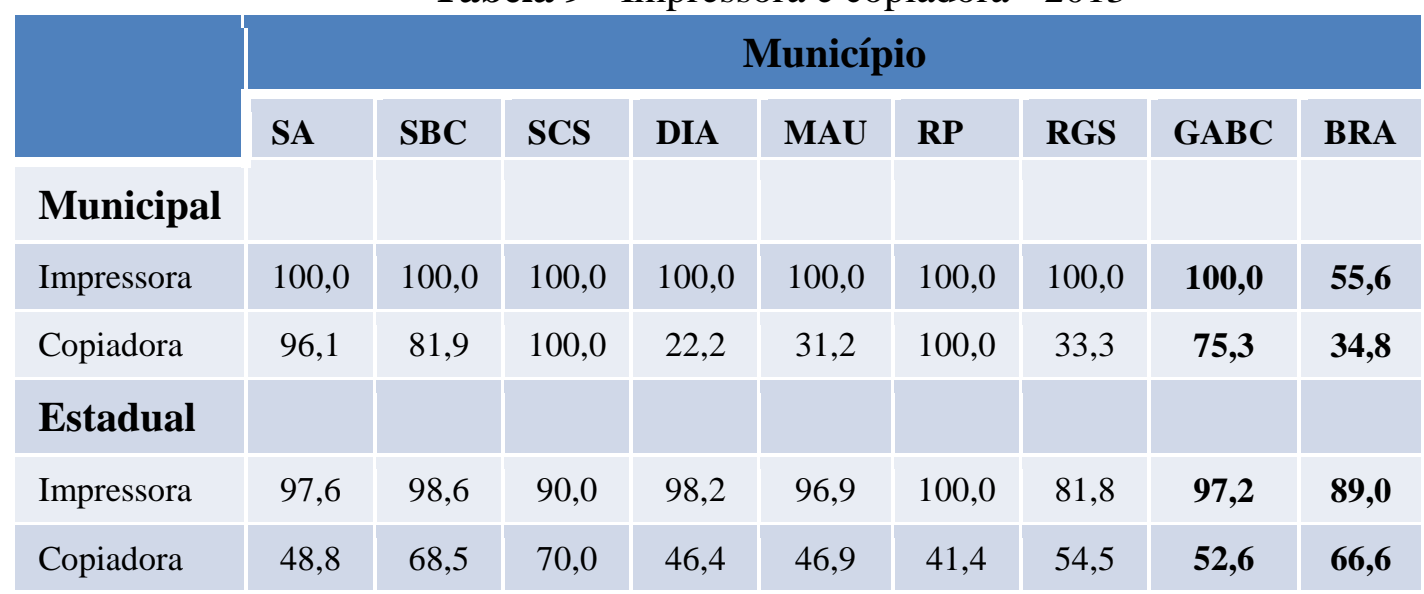

Fonte: Elaboração própria com base nos dados do Censo de 2013 (INEP, 2015). 
Em termos de espaços atrelados à educação especial, sanitário e dependências para deficientes físicos e sala de atendimento especial, a Região vem se fortalecendo, muito embora novos esforços e investimentos sejam necessários para ampliar as ofertas.

Quanto a ter ou não sanitário e dependências para deficientes físicos e sala de atendimento especial, as Tabelas 10 e 11 mostram os resultados:

Tabela 10 - Sanitário para deficientes, sala de atendimento especial e dependência para deficientes físicos - 2013.

\begin{tabular}{|c|c|c|c|c|c|c|c|c|c|}
\hline & \multicolumn{9}{|c|}{ Município } \\
\hline & SA & SBC & SCS & DIA & MAU & $\mathbf{R P}$ & RGS & GABC & BRA \\
\hline \multicolumn{10}{|l|}{ Municipal } \\
\hline $\begin{array}{l}\text { Sanitário para deficiente } \\
\text { físico }\end{array}$ & 76,5 & 86,1 & 45,0 & 44,4 & 25,0 & 55,6 & 0,0 & 66,2 & 19,4 \\
\hline $\begin{array}{l}\text { Sala de atendimento } \\
\text { especial }\end{array}$ & 13,7 & 86,1 & 30,0 & 0,0 & 37,5 & 100,0 & 0,0 & 45,5 & 14,0 \\
\hline $\begin{array}{l}\text { Dependências para } \\
\text { deficientes físicos }\end{array}$ & 43,1 & 41,7 & 30,0 & 25,9 & 6,3 & 0,0 & 0,0 & 33,3 & 15,4 \\
\hline \multicolumn{10}{|l|}{ Estadual } \\
\hline $\begin{array}{l}\text { Sanitário para deficiente } \\
\text { físico }\end{array}$ & 14,3 & 30,1 & 20,0 & 12,5 & 23,4 & 27,6 & 18,2 & 20,8 & 41,5 \\
\hline $\begin{array}{l}\text { Sala de atendimento } \\
\text { especial }\end{array}$ & 17,9 & 17,8 & 10,0 & 17,9 & 20,3 & 10,3 & 18,2 & 17,4 & 26,8 \\
\hline $\begin{array}{l}\text { Dependências para } \\
\text { deficientes físicos }\end{array}$ & 10,7 & 20,5 & 40,0 & 16,1 & 17,2 & 17,2 & 18,2 & 16,8 & 34,5 \\
\hline
\end{tabular}

Fonte: Elaboração própria com base nos dados do Censo de 2013 (INEP, 2015).

Em relação à quadra esportiva, importante espaço para a prática da Educação Física e para a realização de exercícios físico, a região do Grande $\mathrm{ABC}$ tem bons números quando olhamos os dados do Brasil. A Tabela 11 mostra os resultados:

Tabela 11 - Quadra esportiva - 2013.

\begin{tabular}{|l|l|l|l|l|l|l|l|l|l|}
\hline & \multicolumn{3}{|l}{ Município } \\
& SA & SBC & SCS & DIA & MAU & RP & RGS & GABC & BRA \\
\hline Municipal & 88,2 & 86,1 & 90,0 & 48,1 & 56,3 & 33,3 & 0,0 & $\mathbf{7 5 , 8}$ & $\mathbf{2 2 , 8}$ \\
\hline Estadual & 97,6 & 90,4 & 100,0 & 85,7 & 87,5 & 100,0 & 81,8 & $\mathbf{9 1 , 7}$ & $\mathbf{6 7 , 1}$ \\
\hline
\end{tabular}

Fonte: Elaboração própria com base nos dados do Censo de 2013 (INEP, 2015). 
Em uma análise, situando a Região na escala desenvolvida por Soares et al. (2013), é possível verificar a situação privilegiada do Grande ABC. A Tabela 12 sintetiza as análises:

Tabela 12 - Nível da Infraestrutura do Grande ABC e Brasil -2013

\begin{tabular}{|c|c|c|c|c|c|c|c|c|c|c|}
\hline \multirow{2}{*}{ Matrículas } & & \multicolumn{9}{|c|}{ Município } \\
\hline & & SA & SBC & SCS & DIA & MAU & $\mathbf{R P}$ & RGS & GABC & BRA \\
\hline Municipal & E.F. I e II & $83 \%$ & $88 \%$ & $81 \%$ & $70 \%$ & $69 \%$ & $76 \%$ & $47 \%$ & $81 \%$ & $45 \%$ \\
\hline Estadual & E.F. I e II & $76 \%$ & $77 \%$ & $78 \%$ & $72 \%$ & $74 \%$ & $73 \%$ & $68 \%$ & $75 \%$ & $72 \%$ \\
\hline \multicolumn{6}{|c|}{ Até 50\%: Infraestrutura Elementar } & \multicolumn{5}{|c|}{$50 \%$ a 59\%: Infraestrutura Elementar } \\
\hline \multicolumn{11}{|c|}{ 60\% a 69\%: Infraestrutura Adequada } \\
\hline
\end{tabular}

Fonte: Elaboração própria com base nos dados do Censo de 2013 (INEP, 2015).

\section{Considerações finais}

Este estudo trouxe contribuições, revelando a infraestrutura (instalações, equipamentos e os serviços necessários para garantir o funcionamento da escola e auxiliar a aprendizagem do aluno) das escolas de ensino fundamental da Região do Grande ABC Paulista. A compreensão desse cenário pode favorecer a reflexão sobre possíveis alternativas para a organização ou reformas do sistema educacional ou das escolas, considerando que a IE impacta positivamente o rendimento dos alunos em qualquer uma das cinco regiões brasileiras.

Uma questão que a Região tem de avançar relaciona-se aos laboratórios de ciências. A realidade dos municípios neste quesito é pior que a do estado. Já os dados do Brasil mostram uma situação muito mais agravada e enfrentada pelas escolas. Outro incomodo associa-se ao número de bibliotecas. Na esfera estadual, a Região está desprovida desses espaços e novamente os resultados brasileiros são catastróficos. No entanto, é preciso avançar, pois estudos já sinalizaram que esses espaços são relevantes para o desempenho do aluno (ESPÓSITO, DAVIS; NUNES, 2000; MENEZES FILHO, 2007; BIONDI; FELÍCIO, 2007). 
É preciso considerar, entretanto, que a presença do espaço pode não acrescentar nada na aprendizagem dos alunos. A utilização desses locais e recursos torna-se relevante quando associados com iniciativas e projetos pedagógicos. A infraestrutura é um elemento fundamental para a formação do jovem quando são articuladas as instalações, os equipamentos, os serviços, as iniciativas educativas com os projetos pedagógicos e a formação do estudante.

Enquanto o acesso à internet na esfera municipal na Região do $\mathrm{ABC}$ está bem consolidado, no Brasil esta realidade é bem diferente. Somente 29,4\% dos estudantes têm acesso à internet. Considerando que estudos (MENEZES FILHO, 2007; BIONDI; FELÍCIO, 2007) já mostraram que o acesso à internet impacta, positivamente, o desempenho dos alunos, esta presente pesquisa mostra a difícil realidade de aprendizagem vivida pela maioria dos jovens brasileiros.

Os dados mostraram também que ambas, a Região e a realidade brasileira, têm de melhorar em relação aos espaços atrelados à educação especial, sanitário e dependências para deficientes físicos e sala de atendimento especial. Tal situação, entre outras, tem de ser fortalecida para que a escola não seja uma fonte de exclusão.

Uma conclusão válida para este estudo, tomando-se como base a escala desenvolvida por Soares et al (2013), é que na região do Grande ABC a infraestrutura escolar está bem consolidada, superando de longe a dura realidade brasileira. A infraestrutura da Região está localizada no nível avançado (74\%). Tal situação indica uma realidade bem diferente da brasileira. Mas sabemos que a infraestrutura é tributária dos projetos pedagógicos da escola e das ações de ensino dos professores. Desta forma, os resultados são maiores na formação de nossas crianças e jovens.

INFRASTRUCTURE OF BASIC EDUCATION SCHOOLS IN THE REGION OF ABC PAULISTA 
ABSTRACT: The system of educational evaluation is trying to understand the factors that impact student performance on standardized tests, and use such understanding to launch policies to improve education in Brazil. One of these factors is the school infrastructure that in the Brazilian scenario influences student learning. This research explored the infrastructure of schools in the city with the largest Brazilian' IDHM. A case study was conducted by collecting information from schools (fundamental education) in order to reveal a reality that escapes from quantitative studies. The results showed that the infrastructure is suitable for both school functioning and helping student learning, and have some differences among them. All classrooms had a computer, electronic whiteboard and data show, and $70 \%$ had a special room for inclusive education with specialized professionals assisting students. Understanding this reality can foster reflection on possible alternatives for the organization or reforms of the educational system.

KEYWORDS: Infrastructure. Schools. Fundamental Education.

\section{REFERÊNCIAS}

ALBERNAZ, A.; FERREIRA, F. H. G.; FRANCO, C. Qualidade e equidade na educação fundamental brasileira. Rio de Janeiro: PUC-Rio, 2002. (Texto para Discussão, 455.).

BARBOSA, M. E. F.; FERNANDES, C. A escola brasileira faz diferença?: uma investigação dos efeitos da escola na proficiência em matemática dos alunos da $4^{\mathrm{a}}$ série. In: FRANCO, C. (Org.). Avaliação, ciclos e promoção na educação. Porto Alegre: Artmed, 2001. p.121-153.

BIONDI, R. L.; FELÍCIO, F. Atributos Escolares e o Desempenho dos Estudantes: uma Análise em Painel dos Dados do SAEB. Brasília: INEP, 2007.

BRASIL. Lei de Diretrizes e Bases da Educação Nacional. Lei n. 9.394, de 20 de dezembro de 1996. Estabelece as diretrizes e bases da educação nacional. Diário Oficial da União, Brasília, DF, 20 dez. 1996. Disponível em: <http://www.planalto.gov.br/ccivil_03/leis/19394.htm>. Acesso em: 21 fev. 2015.

. Presidência da República. Constituição (1988). Constituição da República

Federativa do Brasil. 1988. Disponível em:

<http://www.planalto.gov.br/ccivil_03/constituicao/constituicao.htm>. Acesso em: 21 abr. 2013.

CÉSAR, C.; SOARES, J. Desigualdades acadêmicas induzidas pelo contexto escolar. Revista Brasileira de Estudos de População, Campinas, v.18, n.1/2, p.97-110, 2001.

CASTRO, C. M.; FLETCHER, P. A escola que os brasileiros frequentaram em 1985. Rio de Janeiro: Ipea, Iplan,1986. 
ESPÓSITO, Y.L.; DAVIS, C.; NUNES, M. M. R. Sistema de Avaliação do Rendimento Escolar: o modelo adotado pelo estado de São Paulo. Revista Brasileira de Educação, Rio de Janeiro, n.13, p.25-53, 2000.

FELICIO, F.; FERNANDES, R. O efeito da qualidade da escola sobre o desempenho escolar: uma avaliação do ensino fundamental no Estado de São Paulo. In:

ENCONTRO NACIONAL DE ECONOMIA, 33., 2005, Natal, RN. Anais... Natal, 2005.

FERRÃO, M. E.; BELTRÃO, K.; SANTOS, D. Políticas de não-repetência e qualidade da educação: evidencias obtidas a partir da modelagem dos dados da $4^{a}$ série do SAEB99. Estudos em Avaliação Educacional, São Paulo, n.26, 2002.

FLETCHER, P. À procura do ensino eficaz. PNUD/MEC/SAEB, 1997. Relatório de pesquisa.

FRANCO, C.; BONAMINO, A. A pesquisa sobre características de escolas eficazes no Brasil: breve revisão dos principais achados e alguns problemas em aberto. Revista Educação On-line, Rio de Janeiro: PUC-Rio, n.1, 2005. Disponível em: <http://www.maxwell.vrac.puc-rio.br/7378/7378.PDF>. Acesso em: 26 fev. 2015.

FRANCO, C.; SZTAJN, P.; ORTIGÃO, M. I. Mathematics teachers, reform, and equity: results from the Brazilian National Assessment. Journal for Research in Mathematics Education, Reston, Virginia, v.38, n.4, p.393-419, 2007.

FULLAN, M. The principal: three Keys to maximizing impact. San Francisco: Jossey-Bass, 2013.

HATTIE, J. Visible learning: a synthesis of over 800 meta-analyses relating to achievement. London; New York: Routledge, Taylor \& Francis, 2009.

INSTITUTO BRASILEIRO DE GEOGRAFIA E ESTATÍSTICA [IBGE].

Infográficos: evolução populacional e pirâmide etária. Disponível em:

<http://www.cidades.ibge.gov.br/painel/populacao.php?lang=\&codmun=354880\&searc $\mathrm{h}=$ sao-paulo|sao-caetano-do-sul|infograficos:-evolucao-populacional-e-piramideetaria>. Acesso em: 26 fev. 2015.

INSTITUTO NACIONAL DE ESTUDOS E PESQUISA [INEP]. Microdados para Download. Disponível em: <http://portal.inep.gov.br/basica-levantamentos-acessar〉. Acesso em: 18 de out. 2015.

\section{LEE, V.; FRANCO, C.; ALBERNAZ, A. Quality and equality in Brazilian}

secondary schools: a multilevel cross-national school effects study. San Diego, 2004. Paper presented at Annual Meeting of the American Educational Research Association. Disponível em: <http://epge.fgv.br/files/2131.pdf>. Acesso em: 26 fev. 2015.

MENEZES FILHO, N. Os Determinantes do desempenho escolar do Brasil. São Paulo: Instituto Futuro Brasil: IBMEC, 2007. 
SÁTYRO, N.; SOARES, S. A infraestrutura das escolas brasileiras de ensino fundamental: um estudo com base nos censos escolares de 1997 a 2005. Brasília: Ipea, 2007. (Textos para Discussão, 1267.).

SOARES, J. F. Qualidade e equiidade na educação básica brasileira: a evidência do SAEB-2001. Archivos Analíticos de Políticas Educativas, v.12, n. 38, 2004.

Disponível em: 〈http://epaa.asu.edu/ojs/article/view/193/319>. Acesso em: 26 fev. 2015.

SOARES, S.; RAZO, R.; FARIÑAS, M. Perfil estatístico da educação rural: origem socioeconômica desfavorecida, insumos escolares deficientes e resultados inaceitáveis. In: BOF, A. M. (Org.). A educação no Brasil rural. Brasília: Instituto Nacional de Estudos e Pesquisas Educacionais Anísio Teixeira, 2006. p.47-68.

SOARES NETO, J. J. et al. Uma escala para medir a infraestrutura escolar. Estudos em Avaliação Educacional, São Paulo, v.24, n.54, p.78-99, jan./abr. 2013. 